Cahiers d'études italiennes

$3 \mid 2005$

NOVECENTO... E DINTORNI

Images littéraires de la société contemporaine

\title{
Conflits armés et conflit intérieur dans Guerra di infanzia e di Spagna de Fabrizia Ramondino
}

Maryline Maigron

\section{OpenEdition}

\section{Journals}

Édition électronique

URL : http://journals.openedition.org/cei/283

DOI : $10.4000 /$ cei.283

ISSN : 2260-779X

\section{Éditeur}

UGA Éditions/Université Grenoble Alpes

\section{Édition imprimée}

Date de publication : 15 juin 2005

Pagination : 155-165

ISBN : 978-2-84310-066-6

ISSN : $1770-9571$

\section{Référence électronique}

Maryline Maigron, «Conflits armés et conflit intérieur dans Guerra di infanzia e di Spagna de Fabrizia Ramondino », Cahiers d'études italiennes [En ligne], 3 | 2005, mis en ligne le 15 décembre 2006, consulté le 26 mars 2021. URL : http://journals.openedition.org/cei/283 ; DOI : https://doi.org/ $10.4000 /$ cei.283 


\title{
CONFLITS ARMÉS ET CONFLIT INTÉRIEUR DANS GUERRA DI INFANZIA E DI SPAGNA DE FABRIZIA RAMONDINO
}

\author{
Maryline Maigron \\ Université de Savoie - Chambéry
}

C'est le portrait de Maya enfant qui illustre la couverture du livre de Fabrizia Ramondino. Maya, la fille de celui qui peignit Guernica. Ce choix iconographique de l'auteur ${ }^{1}$ peut nous guider. Guerra di infanzia e di Spagna raconte l'histoire d'une autre enfant, Titita, dont le père fut consul d'Italie à Majorque pendant la guerre civile espagnole. Une guerre et un père que Titita regarde de ses yeux d'enfant, avec un oil plus tourné audedans d'elle-même et l'autre bien tourné vers l'extérieur, comme Maya (dont les yeux regardent de deux côtés différents). Dans Guerra di infanzia $e$ di Spagna, paru en 2001, les deux conflits armés qu'ont été la guerre civile espagnole et la Seconde Guerre mondiale ne constituent pas une toile de fond : ces deux conflits armés, mais dans une plus grande mesure la guerre civile espagnole (le titre est d'ailleurs explicite), sont au cœur de la vie de Titita et des intentions narratives de l'auteur. Titita naît avec la guerre. Ne fait-elle pas, alors qu'elle est encore bébé, comme le lui racontera Dida, sa nourrice majorquine, le voyage d'un côté de la Méditerranée à l'autre dans un navire de guerre ${ }^{2}$ ? Si elle vient à Majorque avec sa famille, c'est parce que l'Italie mussolinienne participe à la guerre civile espagnole et que l'île est la principale base navale et aérienne des franquistes et de leurs alliés italiens et allemands. Comme Naples, d'où elle vient, et

1. Fabrizia Ramondino nous a confirmé qu'elle avait elle-même choisi ce tableau de Picasso : Maya à la poupée (1938, conservé au Musée Picasso de Paris).

2. Fabrizia Ramondino, Guerra di infanzia e di Spagna, Turin, Einaudi, 2001, p. 69. 
Majorque, où elle vivra de 1937 à 1943, Titita vivra sa guerre. Nous montrerons que les deux conflits armés se transforment en un conflit intérieur, la guerra d'infanzia évoquée par le titre, qui contribue à la formation de la conscience de Titita, la narratrice hérö̈ne. Titita, c'est aussi Fabrizia. En effet, la similitude entre l'enfance de Titita et celle de Fabrizia Ramondino peut être établie par des éléments que l'auteur elle-même a livrés à ses lecteurs, en particulier dans In viaggio, paru en 1995, et cette similitude réside justement dans le fait que Fabrizia Ramondino a vécu à Majorque les premières années de sa vie au moment de la guerre d'Espagne, car son père y était consul d'Italie. Certes, dans Guerra di infanzia e di Spagna, Fabrizia Ramondino fait de Titita un personnage de fiction et gomme les traces biographiques les plus apparentes de sa propre enfance en donnant, par exemple, au père de Titita un nom fictif (Luigi Ferdinando Baldaro ${ }^{3}$ ) et non celui de son propre père (c'est-à-dire Ferruccio Ramondino ${ }^{4}$ ). Cependant, en comparant Titita à la Dulcinée de don Quichotte ${ }^{5}$, elle invite son lecteur à penser que si Titita est une créature inventée, elle peut aussi, dans une sorte de renversement par rapport à Cervantes (chez qui Dulcinée existe mais est aussi une invention de don Quichotte), exister (ou avoir existé) vraiment. De plus, elle dissémine dans le livre lui-même des indices biographiques qui n'échappent pas au lecteur, à commencer par le nom de son personnage. Comme nous l'explique Titita ${ }^{6}$, ce nom, c'est sa façon à elle, enfant, de dire Fabrizia. Dans Storie di patio, la petite fille, que l'on peut considérer comme une première ébauche de Titita, s'appelle Nuria ${ }^{7}$; dans In viaggio, l'enfant n'est pas nommée mais le récit est à la première personne : la dimension autobiographique du récit de l'enfance à Majorque se fait donc, d'un livre à l'autre, plus explicite. Ce double statut de Titita, personnage et personne, apparaît bien dans le traitement narratif de la différence d'âge et d'expérience entre le je narrant et

3. Un nom qu'elle a déjà utilisé dans Storie di patio paru en 1983, mais pour un personnage différent de celui du consul d'Italie (ce dernier apparaît dans le livre, dans un récit intitulé La signora di Son Batle, mais il s'appelle Attilio Mosca).

4. Dans le chapitre d'ouverture, où est racontée l'histoire de la dame de Son Batle, propriétaire de la première maison de la famille dans l'île, l'auteur (la voix narrative est explicitement la sienne) explique qu'elle tient le récit de la dame de Son Batle de la fille même du consul : Fabrizia Ramondino laisse donc supposer que, dans la suite de son livre, la narratrice sera cette dernière et non elle-même.

5. En exergue elle place cette réflexion de don Quichotte : "Dio sa se c’è o no Dulcinea al mondo, e se è immaginaria o non è immaginaria. Queste non sono cose la cui indagine può spiegarsi fino in fondo ». Cervantes, Don Chisciotte della Mancia, libro II, cap. XXXII.

6. "Per la nota difficoltà dei bambini a pronunziare la $f$ e la $z$, avrei potuto trasformare il mio nome o i nomignoli che lo celavano, assai più verosimilmente in Tabita [...] ». F. Ramondino, Guerra di infanzia e di Spagna, op. cit., p. 48.

7. Elle apparaît dans trois récits intitulés I servi, Gli uccelli di Narcís, Il fidanzamento ; dans ces récits, le père de Nuria s'appelle Torquat. 
le je narré. Fabrizia Ramondino conserve la singularité des perceptions du je narré qui est une enfant mais, lorsqu'elle rend compte des difficultés du je narrant aux prises avec l'écriture, elle laisse percer, à mon sens, une difficulté qui est la sienne par rapport à son propre passé. Si donc nous ne suivrons pas le conseil de don Quichotte (" non sono cose la cui indagine può spiegarsi fino in fondo » dit-il à la duchesse à propos de l'existence de sa Dulcinée), ce à quoi d'ailleurs Fabrizia Ramondino nous invite en citant Cervantes, et si nous élargissons à la biographie de Fabrizia notre enquête sur Titita, c'est parce qu'il s'agit pour nous d'observer le rapport entre l'enfant Titita/Fabrizia et les événements historiques, en particulier la guerre d'Espagne. De plus, la guerre civile espagnole étant si peu présente dans la littérature italienne contemporaine, il nous semble important de considérer ce livre et de le lire comme un écho de l'expérience singulière de Fabrizia Ramondino. Nous pensons, en effet, qu'à l'instar du personnage de L'antimonio de Leonardo Sciascia, la guerre d'Espagne a joué un rôle primordial dans la formation de la conscience historique de Fabrizia Ramondino. La guerra d'infanzia qui se livre en Titita n'a pas comme unique origine les conflits armés. Dans le monde de Titita d'autres contrastes, comme par exemple celui qui existe entre sa mère et sa nourrice, aiguisent la connaissance de soi. Cependant, et ce sera notre hypothèse de lecture, c'est le conflit intérieur provoqué par la guerre qui semble avoir perduré chez Fabrizia Ramondino depuis l'enfance : il est donc important de s'interroger sur la fonction que revêt l'écriture même de ce livre à soixante ans de distance.

L'origine du conflit intérieur est liée à l'âge de l'héroïne au moment des conflits armés, et à sa famille. Cette origine est présentée en ouverture du deuxième chapitre : "Quando giungemmo nell'isola, alla fine di febbraio, i mandorli erano già in fiore e il bianco delle corolle si mescolava a quello delle ossa nude nelle campagne. In previsione del nostro arrivo, infatti, avevano ucciso tutti i nostri nemici ${ }^{8} "$. Observons ces phrases du point de vue de l'instance narrative. Le « nous " comme sujet de l'énonciation n'est utilisé que dans ce chapitre, la narratrice utilisera ensuite le « je ». Ce " nous», qui représente la famille de Titita, l'inclut également. C'est ce dernier aspect qui nous intéresse particulièrement. Lorsque, bébé, Titita arrive à Majorque, la guerre a déjà commencé dans l'île : les " ossa nude " renvoient aux massacres perpétrés, dès le mois d'août 1936, par les phalangistes avec l'aide militaire de l'Italie ${ }^{9}$, et l'ennemi est déjà désigné. En

8. F. Ramondino, Guerra di infanzia e di Spagna, op. cit., p. 21.

9. C'est sous le commandement d'Arconovaldo Bonaccorsi, appelé le conte Rossi, ancien chef des 
effet, dans l'expression "nos ennemis ", le possessif marque bien que l'appartenance de Titita à la famille du consul d'Italie la situe d'emblée dans " un camp ": son père représente un pays qui est engagé dans la guerre civile espagnole aux côtés des franquistes. De plus, ce "nous " illustre le fait que, compte tenu de son âge au moment des conflits, la conscience historique et politique de Titita n'est pas encore formée : "le camp " auquel sa famille appartient est donc, deux fois, un camp qu'elle n'a pas choisi. Il faut souligner également que pour Titita la différence entre la vie publique et la vie privée de son père est difficilement perceptible. Ainsi, par exemple, dans sa maison, Titita côtoie des aviateurs italiens. Le « nous " utilisé dans ce chapitre est également l'expression du je narrant dont la conscience historique transparaît, par exemple, lorsqu'il est question de « la suadente conquista dell' isola che si tramava nella nostra casa ${ }^{10}$ ”.

Observons, maintenant, pourquoi Titita vivra cette appartenance familiale comme un conflit. Le récit d'un épisode appelé la farce de la mayonnaise (Fabrizia Ramondino choisit le mot beffa) peut servir de point de départ. Lors d'un repas privé donné dans sa maison, le consul décide de fêter l'amitié italo-espagnole : "Il clou di quel pranzo doveva essere un'insalata russa sopra un pesce lungo cinque metri. E sul manto di maionese che la ricopriva doveva campeggiare in rosso, composta da striscioline di barbabietola, la scritta : Amistad Italo-española ». Mais, lorsque le plat est servi, les sourires du consul et de ses invités se figent car l'inscription qui trône sur le poisson est : "Viva la Republica » (ibid., p. 27). L'énonciation est importante dans ce passage. En effet, l'épisode de la beffa s'étant produit peu après l'arrivée de la famille à Majorque, le récit ne peut pas être un souvenir direct de Titita. En réalité, la beffa lui a été racontée : «Orbene, poco dopo il nostro arrivo, avvenne la Beffa della maionese di cui ancora si favoleggia a Maiorca " (ibid., p. 26). En plaçant l'évocation de cet épisode dès le deuxième chapitre du livre, l'auteur signifie que Titita grandit également au contact de Majorquins qui ont organisé la beffa (et, ou, en ont ri) et pour lesquels l'amitié italo-espagnole célébrée chez le consul n'est pas une fête. De plus, Titita entretient un contact direct avec la population de l'île. En effet, elle apprend et parle le majorquin avec sa nourrice Dida et les serviteurs de sa maison, avec son compagnon de jeux, Paco. Et sa connaissance de la langue majorquine (une langue que Fabrizia

squadristi de l'Émilie Romagne entre 1920 et 1922, que les phalangistes se livrent à une répression féroce sur l'île.

10. F. Ramondino, Guerra di infanzia e di Spagna, op. cit., p. 26. 
Ramondino retrouve et restitue dans ce livre en un effort de mémoire où la dimension biographique est, encore une fois, perceptible ${ }^{11}$ ) lui donne accès à des informations que ses parents ne lui communiquent pas (Titita dit de son père : "Papito infatti aveva ciglia lunghe lunghe, e aveva sempre una qualche verità da nascondere ${ }^{12}$ ") ou n'ont pas eux-mêmes ("Basta chiedere ai nostri ragazzi per sapere cosa pensa la gente di noi Italiani » [ibid., p. 263], dit son père) : le majorquin l'ouvre donc à la connaissance d'un autre aspect des événements. Les insulaires font exister pour elle cet autre "camp » que sa famille désigne comme celui des ennemis et le lecteur pressent que la remise en question de l'amitié italo-espagnole sera au centre du conflit intérieur vécu par Titita.

Le conflit intérieur est également aiguisé par le fait que les adultes de la famille sont eux-mêmes divisés sur la question de la guerre. Il existe, en effet, une opposition très forte entre le père et la grand-mère maternelle, d'une part, et, dans une moindre mesure, entre le père et la mère. Titita associe son père à la guerre (et aux tueries) : " Pareva si nutrisse di carogne » (ibid., p. 195), dit-elle, pour décrire sa façon de le percevoir. Il convient de souligner que le père ne produit pas de discours sur la guerre. Cependant, si l'on examine un passage (dans un chapitre intitulé " Notizie della guerra ») où la narratrice passe de l'évocation de la Voix de la radio (la voix de Mussolini, qui remercie ses combattants) à la voix de son père qui lit les titres des journaux, il apparaît clairement que Titita ne comprend pas encore le sens des événements mais qu'elle associe son père au discours fasciste officiel ${ }^{13}$ (ibid., p. 366-367). À l'opposé, il y a sa grand-mère maternelle, qui se prononce nettement contre la guerre : elle parle de barbarie ${ }^{14}$, de mensonges à propos du discours des autres adultes sur la guerre (" quando dicono cose belle della guerra, mentono " [ibid., p. 197], ditelle à Titita) ; cette grand-mère, qui vit à Naples, a une expérience directe de la Seconde Guerre mondiale, elle évoque les morts de Naples et met en parallèle différents conflits armés (elle lui parle aussi de la Première Guerre mondiale). La mère, enfin, a une attitude ambivalente dans la mesure où, pour Titita, elle semble osciller entre son statut d'épouse du consul qui se doit de recevoir chez elle les aviateurs italiens (ce qui, nous le verrons, représente pour Titita une douloureuse réalité) et une position beaucoup plus critique vis-à-vis, en particulier, de la guerre civile espagnole. En effet,

11. Dans In viaggio, en 1995, donc ( $c f$. infra, note 15), Fabrizia Ramondino disait qu'elle ne comprenait plus cette langue.

12. F. Ramondino, Guerra di infanzia e di Spagna, op. cit., p. 154.

13. F. Ramondino, Guerra di infanzia e di Spagna, op. cit., p. 366-367.

14. "Mamita parlava di sacrifici e la nonna di barbarie ", Guerra di infanzia e di Spagna, op. cit., p. 95 . 
à un certain moment, la narratrice rapporte que sa mère lit Les Grands Cimetières sous la lune de Georges Bernanos (un livre que lui a envoyé son cousin de Naples). Il n'est pas surprenant que sa mère, qui est présentée comme une femme très cultivée, lise ce livre si tôt (ce livre a paru en France en 1938), mais il est frappant qu'elle passe d'une conversation de salon avec les auteurs des tueries à la lecture d'un livre qui dénonce les massacres des phalangistes à Majorque. De toute évidence, la singularité d'une telle lecture ne peut avoir été saisie qu'a posteriori par Titita/Fabrizia : l'évocation de cette lecture par le je narré a donc pour but de souligner que tout ce qui touche aux conflits armés apparaît comme contradictoire à l'enfant.

Il est aisé de comprendre que la nature première du conflit intérieur vécu par Titita est affective. Titita se sent déchirée (en italien nous dirions que "Titita è combattuta ", une expression qui rend bien l'idée de conflit que nous avons retenue) entre la position et le rôle des adultes par rapport à la guerre et l'affection qu'elle leur porte. Certaines images du père rendent bien cette déchirure, comme celle-ci : «Gli occhi di Papito erano gremiti di ombre che ne trasportavano lontano, in una cosa che si chiamava « la guerra », l'espressione carezzevole » (ibid., p. 210).

Le conflit intérieur se présentera principalement sous la forme d'une interrogation qui peut être formulée ainsi : les ennemis ou les amis sont-ils ceux qui sont désignés comme tels par les parents ? Un épisode permet de mettre en lumière cet aspect. Un jour où Titita se trouve dans un champ de blé, un bombardier surgit dans le ciel et pique :

A un tratto ci fu un rombo terribile sopra la mia testa. Il cielo si oscurò. Tutta la vita che fremeva nel campo volò via o si appiatì contro la terra. Moscerini mi accecarono gli occhi. Sul collo mi parve di avvertire un colpo d'ala : non un fremito, un taglio. Era un aereo. Con l'ala aveva quasi sfiorato le spighe. (Ibid., p. 227)

Terrorisée, Titita revient à la maison et, lorsque sa mère rentre un moment plus tard, elle lui dit : "Sai quei piloti sono nostri amici " (ibid., p. 228). Cette réflexion de sa mère représente à la fois un déni de l'expérience qu' elle vient de vivre (ce pilote, qui a failli me tuer, me veut du bien car c'est un ami de ma famille) et une dénégation du sens du mot ami (un ami serait quelqu'un qui veut me tuer). Titita perçoit donc comme une contradiction le commentaire maternel de son expérience. Un conflit intérieur que Titita exprimera, à sa façon d'enfant, c'est-à-dire sous la forme d'une perception (et non de façon idéologique), en remarquant, par exemple, dans un autre passage du livre, que l'étoffe des uniformes des aviateurs italiens pique ses jambes quand ceux-ci la prennent dans leurs bras lors des réceptions de ses parents (ibid., p. 90). Il apparaît dans l'épi- 
sode du champ de blé que les sens de Titita ne mentent pas, alors que les adultes peuvent être aveuglés par l'idéologie. C'est cette vision non idéologique de l'Histoire propre aux enfants (une vision qui renvoie aux personnages d'Italo Calvino, d'Elsa Morante) qui est au cœur du conflit intérieur vécu par Titita.

L'apprentissage du sens des mots est donc pour Titita une expérience douloureuse car la position diplomatique de son père et le contexte politique des deux guerres la place au centre des différentes alliances que l'Italie noue. Chaque nouveau conflit armé semble introduire son sens quand les amis d'hier, et même les amis de la vie privée, deviennent des ennemis et inversement. Ainsi, au début de la Seconde Guerre mondiale, son père déclarera à propos d'une famille hollandaise qu’ils fréquentaient : "Ora che l'Italia è entrata in guerra, dovremo troncare i rapporti con i Van Loo. Perché domandai. Perché il loro paese non è un nostro alleato " (ibid., p. 89) ; inversement, dans In viaggio, Fabrizia Ramondino raconte qu'au moment de leur départ de l'Espagne, c'est-à-dire après l'armistice, son père reproche à sa mère de saluer des Allemands ${ }^{15}$.

D'où une perception accrue, chez Titita, du contraste entre l'utilisation du mot guerre par les adultes et leurs actes. Ainsi, Titita ne supporte pas l'apparente insouciance de ses parents vis-à-vis de la guerre : «Parlavano della guerra e sorridevano; e tutte le volte in cui ero presente a queste loro conversazioni fuggivo dalla stanza. Solo con la nonna potevo parlare della guerra, tenendo la faccia nascosta fra le sue gonne ${ }^{16}$ ". Le conflit est donc une étape vers la prise de conscience historique et sa grand-mère, ainsi que l'autre guerre, celle que vit Naples, servent de révélateur : lorsque sa grandmère manifeste son indignation face à la vie mondaine que les parents de Titita conduisent malgré la guerre, elle parle de la souffrance des Napolitains : "Devo tornare a Napoli. Lì la gente soffre, non c’è la spensieratezza che regna in questa casa, dove tutti parlano di guerra come fosse una mano di poker, un divertimento $»$ (ibid., p. 149).

La guerre entre également en conflit avec le monde enchanté de l'île ("L'isola incantata » est le titre du deuxième chapitre) : dans le ciel de l'île les avions de guerre ont remplacé les oiseaux (et quand les hommes deviennent des oiseaux : "cacano merda e bombe e mentono " dit Titita [ibid., p. 197]). La guerre, représentée surtout par les navires et les avions, pénètre dans le monde de Titita et l'altère. La guerre altère le regard de Titita et ses sens. Ainsi, lorsque sa mère l'emmène voir, depuis la terrasse d'un café, des

15. F. Ramondino, In viaggio, Turin, Einaudi, 1995, p. 58.

16. F. Ramondino, Guerra di infanzia e di Spagna, op. cit., p. 83-84. 
navires de guerre qui ont jeté l'ancre dans le port de Palma, Titita remarque : "Nel sapore del gelato mi pareva di avvertire un retrogusto come di ferro [...]" (ibid., p. 116). Par une sorte de contamination, la vue des navires de guerre dans le port provoque une transformation de la saveur agréable de la glace en un goût désagréable ; de même, la vue des uniformes des soldats modifie la perception du monde : ainsi, le biscuit qui accompagne la glace semble perdre sa saveur puisqu' il prend la couleur des treillis militaires ( Il biscotto al pistacchio aveva il colore delle tute mimetiche dei paracadutisti " [ibid., p. 116]). Cette altération rend bien compte du conflit intérieur qui se joue ici : les bateaux de guerre ne sont pas un spectacle pour Titita et leur vision gâche son plaisir d'être enfant. De plus, l'altération se produit à la demande de la mère et Titita perçoit, encore une fois, comme une contradiction l'invitation de celle-ci à savourer une glace au goût de fer. De sorte que tout ce qui est métallique renvoie à la guerre : "Giocavo alla guerra al rumore metallico delle forbici » (ibid., p. 68), et la guerre est une kyrielle de mots de métal ${ }^{17}$. La guerre pénètre dans les jeux de l'enfance:

Scavavo la terra e vi deponevo dentro insetti vivi e morti, i fiori di pisello-lattanti, i rametti spinosi che immaginavo soldati ; giocavo alle tombe. Ispiravano forse quel gioco la gelosia verso i miei fratelli e l'imperversare della guerra intorno a noi. (ibid., p. 41)

Titita et Paco jouent tantôt dans le camp républicain, et combattent contre le " conte Rossi ", tantôt dans l'autre camp en combattant contre le capitaine Bayo ${ }^{18}$. Ces jeux des enfants, qui miment le conflit entre les deux camps, sont à la fois une représentation de la déchirure vécue par Titita (à qui l'on demande d'être d'un côté et non de l'autre) et une expression de sa prise de conscience idéologique (l'existence des deux côtés) ; une prise de conscience progressive, qui s'exprime vraiment à la fin du livre, sur le navire de guerre qui ramène la famille en Italie, après l'armistice, car Titita qui retient la phrase de l'officier anglais à son père : "Lei ha solo, come me, servito il suo paese " (ibid., p. 408), est déjà celle dont la conscience politique s'est formée (le je narré rejoint le je narrant) puisqu' elle pose le problème de la culpabilité.

Deux récits de la guerre d'Espagne ne sont pas le fait de la narratrice mais contribuent à la formation de sa conscience : ce sont le récit de Geronimo et le récit plus bref de Malaquias. Il est aisé de comprendre

17. "La guerra era una sfilza di vocaboli di metallo [...] ", F. Ramondino, Guerra di infanzia e di Spagna, op. cit., p. 366.

18. En août 1936, Bayo avait tenté de débarquer sur l'île, en partant de Barcelone, pour la rendre à la République (F. Ramondino, Guerra di infanzia e di Spagna, op. cit., p. 232). 
pourquoi ces récits sont délégués à d'autres narrateurs : ils racontent des faits advenus dans d'autres lieux, Guernica pour le premier, ou à une autre période, le début des années 1930 pour le second. Considérons le récit de Geronimo dans le chapitre intitulé « Il bambino di Guernica». Geronimo, le narrateur, est un moine franciscain vivant à Majorque mais originaire de Guernica. Geronimo raconte le bombardement de Guernica à travers l'histoire d'un enfant, el nin, qui a été blessé mais qui a survécu grâce à ses soins. Ce méta-récit a des destinataires intradiégétiques, à savoir Titita et Paco, ainsi que Dida et les autres serviteurs de la maison, et sa fonction première est elle-même intradiégétique : il s'agit d'informer sur ce qu'est la guerre. Rappelons qu'en racontant le bombardement de Guernica, advenu en mars 1937, Geronimo raconte un fait de guerre récent. La réaction des serviteurs est à ce titre significative : la présence même de l'enfant (qui est resté sous la protection de Geronimo) ne suffit pas à les convaincre de la véracité des faits rapportés par Geronimo : «A quella storia si commossero tutti, anche se, in verità, non credettero al monaco, benché quel bambino vestito da monacello, senza genitori, muto, era ben lì davanti a loro ; ma non era detto che fosse proprio di Guernica » (ibid., p. 207). Aucune précision n'est donnée sur cette incrédulité mais nous pouvons supposer que celle-ci provient, certes, de la singularité du moine, qui apparât peu conventionnel aux yeux des serviteurs, car il aime la bonne chère, jure, mais également du caractère effroyable, et donc invraisemblable, à leurs yeux, des faits rapportés.

La fonction du récit de Geronimo ne peut pas être pour les destinataires extradiégétiques (les lecteurs que nous sommes) purement informative : les événements rapportés par celui-ci sont bien connus. Ils sont connus mais nous pouvons, aussi, les avoir oubliés. Notre première hypothèse est donc que ce récit revêt une fonction testimoniale. De plus, il convient de s'interroger sur la longue gestation de ce livre dont la première ébauche " remonte au début des années quatre-vingt ${ }^{19}$ ". Comme nous l'avons déjà souligné, une première étape du récit de Majorque est Storie di patio, qui paraît en 1983 ; ensuite, en 1995, Fabrizia Ramondino publie dans In viaggio deux textes repris intégralement ou partiellement dans Guerra d'infanzia e di Spagna ${ }^{20}$. Cette longue maturation est évoquée

19. Titti Marrone, "Occhi di bambina ", interview de Fabrizia Ramondino, Il Mattino, 17 octobre 2001.

20. Il s'agit de Alzati e cammina, repris avec le même titre et de légères modifications, et d'un passage du récit intitulé Guide, repris et développé dans le chapitre intitulé "Ursula »; Fabrizia Ramondino évoque également son enfance à Majorque dans d'autres récits de In viaggio comme, par exemple, dans Veicoli, où elle raconte le retour en Italie de sa famille après l'armistice. 
dans Guerra di infanzia e di Spagna, où le je narrant intervient directement dans la narration (par le passage du passé au présent) pour faire part au lecteur de la difficile reconstruction des fragments épars de son passé. Selon nous, cette difficulté est liée à la fois au caractère biographique du passé qu'il s'agit de reconstruire et à la dimension traumatisante du conflit intérieur provoqué par le contraste déchirant entre le milieu social et idéologique dans lequel Titita (Fabrizia) naît et grandit et les événements historiques auxquels elle est confrontée. Il convient de rappeler, à ce point de notre analyse, une réflexion que Fabrizia Ramondino livrait à ses lecteurs dès 1995 dans In viaggio : "Dopo che ebbi acquisito una relativa consapevolezza storica e politica ", écrivait-elle alors,

decisi di non andare in Spagna finché non fosse caduto il regime franchista [... Non è vero che le colpe dei padri non ricadono sui figli — se a colpa si aggiunge " senso di... ". Prova ne sia quanto è accaduto a tanta gioventù tedesca negli anni Cinquanta e Sessanta. Avevo trascorso la mia prima infanzia, dal'37 al'43, a Maiorca, dove mio padre era stato nominato console ${ }^{21}$.

Tout enfant se sent impliqué dans ce que ses parents font et disent, et le sentiment de culpabilité qu'il peut éprouver sera lié à la gravité des actes de ceux-ci et au degré de son implication (en comparant son sentiment de culpabilité à celui des enfants des nazis, Fabrizia Ramondino semble souligner [et juger] la gravité des actes de ses propres parents). Il ressort de Guerra di infanzia e di Spagna que l'implication de Titita/Fabrizia dans les actes de ses parents est très forte. Sa famille lui demande (Titita n'est pas " je » elle est " nous »), à Majorque en guerre, de ne pas être seulement témoin mais, implicitement, d'être aussi " acteur » et donc coupable (ce que, compte tenu de son âge, elle ne peut être). Comme nous l'avons vu, Titita est tiraillée, ballottée entre le domaine privé de l'affection et le domaine, public, de la violence et de la guerre, de sorte que lui est refusé le seul lieu où elle pourrait n'appartenir à aucun " camp ", à savoir l'enfance : "Ho capito che non si può fuggire in nessun luogo, né sulla luna, né sulla terra ${ }^{22}$ ", dit Titita, pour commenter le moment où le bombardier a piqué, manquant de la tuer. Le conflit intérieur qui était, au moment de l'enfance, l'expression non idéologique de sa révolte contre les actes ou les paroles des adultes, semble avoir perduré depuis l'enfance sous la forme d'un sentiment de culpabilité. Guerra di infanzia e di Spagna apparaît alors comme une tentative de dépassement de ce sentiment de culpabilité. D'une part l'écriture, qui met en lumière le conflit intérieur vécu par l'enfant, revêt une dimension cathartique (cependant, mais ce dernier aspect

21. F. Ramondino, In viaggio, op. cit., p. 93.

22. F. Ramondino, Guerra di infanzia e di Spagna, op. cit., p. 229. 
mérite un approfondissement, nous pouvons nous demander si cette catharsis est vraiment profonde ou si elle est, seulement, de nature idéologique). D'autre part, avec le récit de Geronimo en particulier, Fabrizia Ramondino commémore dans ce livre les morts républicains de la guerre civile espagnole. Ainsi, le fil tendu entre Titita et Maya Picasso pourrait bien exprimer un désir de filiation imaginaire différente : regarder un autre père (idéologiquement intact) pour parler de la guerre d'Espagne. Car le choix de ce portrait de Maya est également, selon moi, un hommage à celui qui peignit le tableau par lequel le monde entier se souvient de la guerre d'Espagne et du massacre perpétré par l'aviation allemande dans la petite ville basque de Guernica. Un massacre auquel Fabrizia Ramondino sauve un enfant, el nin, qui, comme les enfants du tableau de Picasso, nous aide à nous souvenir de Guernica et de la guerre d'Espagne. 